\title{
Virtual Prototype Modeling and Simulation of Pipe Wagon Articulating System
}

\author{
Ying $\mathrm{Li}^{1}$, Samuel Frimpong ${ }^{1}$, Wenyuan Liu ${ }^{2}$ \\ ${ }^{1}$ University of Missouri-Rolla, Rolla, USA \\ ${ }^{2}$ Washington University in St. Louis, St. Louis, USA \\ E-mail: liyinglzh@yahoo.com \\ Received September 9, 2011; revised October 18, 2011; accepted October 25, 2011
}

\begin{abstract}
Virtual prototype of pipe wagon articulating (PWA) system has been developed and simulated based on the kinematics and dynamics of machinery and Automatic Dynamic Analysis of Mechanical Systems (ADAMS) software. It has been integrated with real-time three dimensional (3-D) system simulations for detailed and responsive interaction with dynamic virtual environments. By using this virtual model, the conceptual design examination and performance analysis of the PWA system have been realized dynamically in virtual laboratory. System dynamic force, displacement and tension of pipe have been measured through verifying this 3$\mathrm{D}$ virtual prototype. By comparing the static tension and dynamic tension of pipe, the difference between the two kind tensions has been found. The simulated dynamic tension is much greater than the static tension obtained from the static theory. The results attained in this work suggest that the conceptual designed PWA system can meet the requirements of the operation.
\end{abstract}

Keywords: Pipe Wagon Articulating, Dynamic Modeling, Virtual Prototype, Dynamic Simulation

\section{Introduction}

For efficient and economic extraction and haulage of oil sands from production faces, the "at face slurrying (AFS)" technology is currently being investigated at the research and technology development levels. The AFS technology will be used to create and transport oil sands slurry from production faces through flexible pipeline system to link the existing hydro-transport system. The three options have been proposed to transport oil sands slurry from the face to a fixed pipeline system based on preliminary economic modeling, simulation and analysis of various conceptual models.

The PWA system is one of the three AFS options. A lot of efforts have been invested to conceptualization of the PWA mechanical system and detail numerical modeling for investigation of oil sands multiple-phase problem in pipeline [1-4]. A conceptual design of the PWA system has been proposed in our lab. In this proposal, the PWA system is composed of linkages of pipe wagons connected with flexible pipes. This flexible arrangement accommodates the horizontal and vertical displacements of the mobile system as it follows the hydraulic shovels in the excavation process. In all research, a lot of attention has been given to study the single-phase and multiphase flow of oil sands slurry in PWA flexible pipe. The mechanics of oil sands slurry flow in PWA flexible pipeline system has been formulated and simulated over an extended period [1]. A numerical simulator has been developed to provide numerical solutions of the flow in flexible pipe as a 3-D multiphase problem [2]. However, implementation of the PWA system in real-time and inside a virtual environment, has not been carried out. And, there has not been investigation on system motion and engineering performance analysis of the PWA system so far. The continuing research will examine the handling performance of the system to represent the motions and forces of various components by modeling and simulateing real-word PWA system in a virtual laboratory.

The literature that been reviewed in reference [5] indicates a consistent viewpoint of the virtual prototype modeling using to simulate the ground articulating pipeline (GAP) system. Therefore, this methodology still will be used in the simulation of the PWA system. Mechanical system simulation (MSS) in ADAMS software can be used for ongoing virtual modeling and simulation of the PWA system. MSS can be employed to simulate the motion and force of the PWA systems based on machinery 
kinematics and dynamics. In order to realize dynamic modeling of the PWA system, the kinematics and dynamics models of the system are built in terms of the theory of machines and mechanisms [6,7]. The principles of mechanics [8] can be used to static modeling mechanical system. So, the solved kinematics and dynamics of machinery can yield equations for dynamic-motion, staticforce and dynamic-force analysis [9].

However, for the dynamic simulation of the PWA system two key factors differ from the GAP system. One of the key factors is system kinematics modeling. The pipelines exhibit highly geometrically nonlinear behavior. They are very flexible and undergo large displacements before attaining their equilibrium configuration. Due to this inherently nonlinear behavior, the flexible pipelines do not fit the assumption of rigidity. They usually have no effect on the kinematics of the system but do play a role in supplying forces. The pipelines of the system are usually ignored during kinematic analysis, and their force effects are introduced during dynamic simulation [7]. Another of the key factors is pipeline modeling. The PWA system is made up of linkages of wagons connected by rubber pipelines. The rubber pipelines are used for transmitting forces or displacements between wagons. Because transmission paths are often convoluted, and the pipe performance is dependent on phenomenon such as friction and stretching, it is difficult to model pipelines using standard tools available in most mechanical system simulation packages. The way to model fixable pipeline in $\mathrm{AD}$ AMS is to discretize the pipe into segments. The segments are then attached with a constraint.

In this work, the theoretical modeling and virtual prototype simulation of the PWA have been focused on. Using the virtual model developed in this work, the following work has been carried out: 1) realization of dynamic simulation; 2) creation of 3-D solid visualization models with 3-D motion for the PWA system; 3) determination of important engineering data, such as maximum force necessary to drive the PWA machinery using reality and virtual prototypes; 4) analysis of the distribution of tension along pipe and comparison of static tension with dynamic tension.

\section{Concetual Design of the PWA System}

The PWA system will facilitate the conveyance of oil sands slurry to joint a fixed pipeline or existing hydrotransport train (HTP). This system has been designed and developed to withstand the oil sands mechanical and chemical characteristics and handle oil sands slurry rate or flow rate of $6100 \mathrm{tph}$. It must accommodate production face advance of $60 \mathrm{~m} /$ day or $400 \mathrm{~m} /$ week with a robust system components interfaces. The slurry component sizes must be a fraction of minus $80 \mathrm{~mm}$ with a specific gravity of 1.6. The PWA concept is illustrated in Figure 1(a). This system will work with a shovel, mobile slurry system with slurry pump system, PWA system and fixed pipeline system. The PWA system consists of linkages of wagons connected by FlexRite flexible pipelines. In this combined system the shovel excavates and feeds dry oil sands lumps into the mobile slurry system and with the addition of hot water into the system, oil sands are slurried. The resulting oil sands slurry is then pumped through the PWA system to join the fixed pipeline or existing HTP.

Figure 1(b) indicates that the PWA system will consist of a series of rigid truss frames on castors and will be allowed to swivel relative to each other. Each frame will support concentrated 24" diameter slurry and 18” diameter fresh water lines. A flexible pipeline assembly is required to allow flow of both slurry and fresh water while permitting the position changes between adjacent trusses. FlexRite pipes are more flexible than conventional steel pipes and provide maximum bending with smooth flow of materials. The flow across the FlexRite pipeline can change in any direction due to the flexible nature of the pipeline system. The flexible pipe can bend to a maximum angle of $60^{\circ}$. Two types of particles or mixtures impingement can occur including straight horizontal flexible pipe and bend (from $0^{\circ}$ to $60^{\circ}$ deflection) flexible pipe.

A mining sequence can be established for a movement of a shovel within the pit. The mobile slurry system is designed to follow the shovel as it traverses the pit. During this operation, the pipe will move as they follow the movements of the shovel as illustrated in Figure 1(a). To accommodate a shovel advance, the PWA arms will be added at various advance rates as illustrated. As the mine face advances the stretching PWA will follow shovel. The PWA movement will be automated and robotized through computer vision systems. In this case, the PWA can accumulate or un-accumulate allowing the effective working envelope between the mine face and fixed pipeline to be varied.

\section{Theoretical Models of the PWA System}

\subsection{Motion Model of the PWA Wagon}

The requirement by the PWA system is that of causing an output member to move from one position to another. In analyzing motion, the first and most basic problem encountered is that of defining and dealing with the concepts of position and displacement. Since motion can be thought of as a time series of displacements between successive positions, it is important to analyze exactly the positions of mechanism in the PWA system. Figure 2 is a schematic drawing of the PWA system including the 


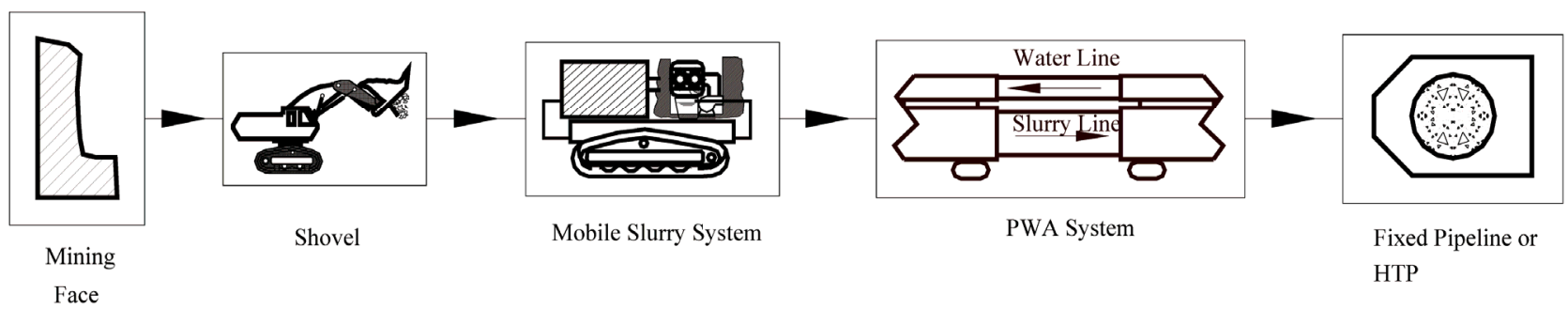

(a)
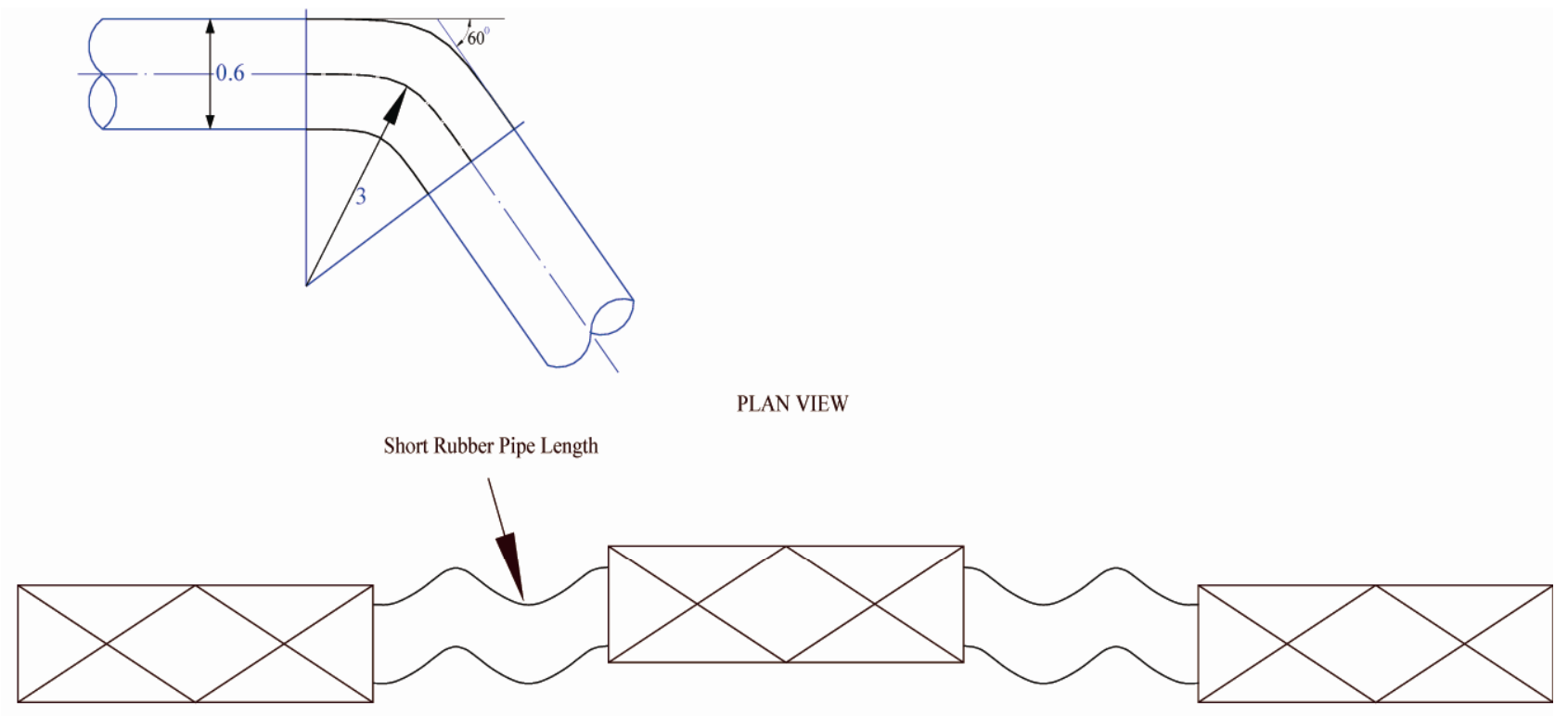

SIDE VIEW

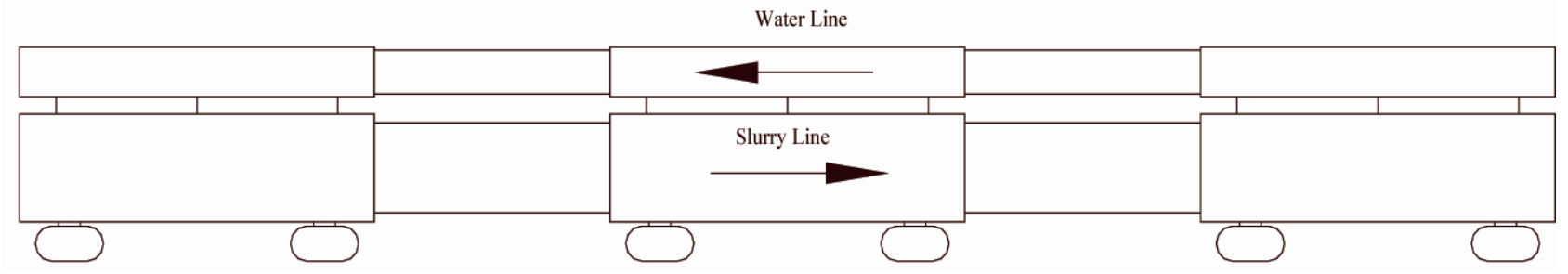

(b)

Figure 1. (a) Conceptual components of the PWA technology [1]; (b) Conceptual views of the PWA system [1].

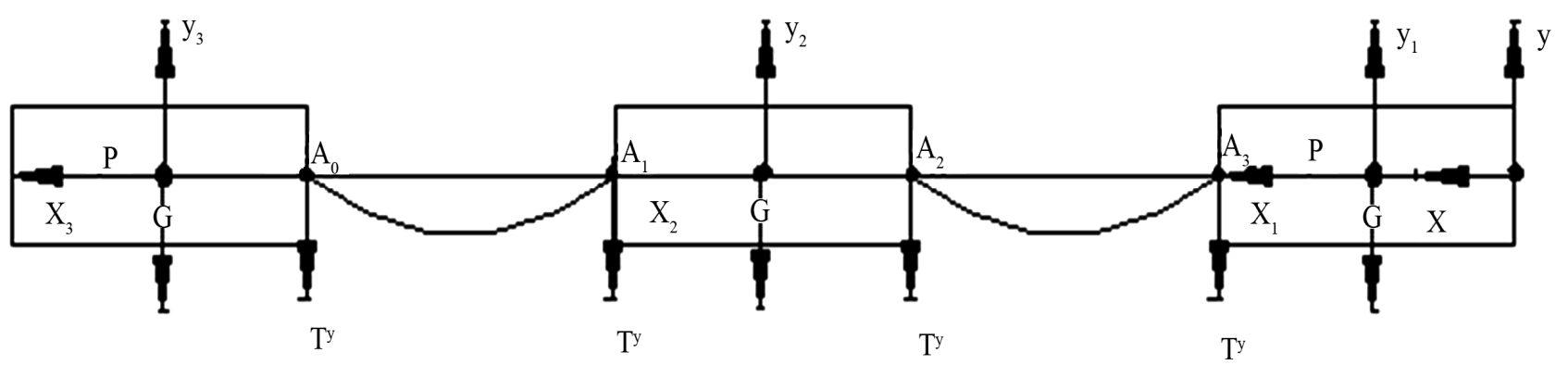

Figure 2. Schematic diagram of the PWA system. 
ground, two pipe units and three wagons. Furthermore, this mechanism is a multi-loop linkage combined of same two or more pipe wagon units. To simplify design, one pipe wagon unit has only been studied. If it is assumed that the velocity of every wagon is constant, the displacement of every wagon is Equation (1).

$$
x_{i}=v_{i} t
$$

where $v_{i}$ is the wagon velocity value, $t$ is the operation time. If the PWA system consists of $N$ pipe wagons, the total displacement of system is Equation (2).

$$
X=\sum_{i=1}^{N} v_{i} t
$$

\subsection{Dynamic Model of the PWA System}

In the PWA system, the desired motions of the mechanisms are specified in advance by production requirement. Even though the wagon is driven at constant speed, this does not mean that all points of the pipelines have constant velocity vectors or even that other parts of the system will operate at constant speeds; there will be accelerations and therefore system with moving parts will not be in equilibrium. Analytical methods for investigating dynamic forces in the PWA system employ mathematical models that are solved for unknown forces associated with known mechanism motion. Solving this problem requires definitions of the actual shapes, dimensions, and material specifications to determine the centers of mass and mass moments of inertia of the parts, which will be given in section 4.0. Then, the Lagrange method of multirigid-body system is used to establish the equations of kinematics and dynamics of the PWA system. The displacement, velocity, acceleration and force can be obtained.

Cartestian coordinate of the center of mass for rigid body $i$ and Euler angle or generalized Euler angle of rigid body are regarded as generated coordinates,

$q_{i}=\{x, y, z, \psi, \theta, \phi\}_{i}^{T}, q=\left\{q_{1}^{T}, q_{2}^{T}, \cdots, q_{n}^{T}\right\}^{T}$. The appropriate dynamic model of the system [8] is given by Equation (3).

$$
\dot{p}_{i}-\frac{\partial L}{\partial q_{i}}+\sum_{j=1}^{m} \lambda_{j} \frac{\partial \Phi_{j}}{\partial q_{i}}-\sum_{k=1}^{n a} F_{k} \frac{\partial r_{k}}{\partial q_{i}}=0
$$

Restriction equations are written as

$$
\begin{gathered}
\dot{p}_{i}-\frac{\partial L}{\partial \dot{q}_{i}}=0 \\
u_{i}-\dot{q}_{i}=0 \\
\Phi_{j}\left(q, t_{n}\right)=0 \\
F_{k}-f_{k}\left(q, u, \lambda, t_{n}\right)=0
\end{gathered}
$$

where $\mathrm{L}$ is Lagrangian $=\mathrm{T}-\mathrm{V}, \mathrm{T}$ is the kinetic energy of system, V is the potential energy of system, $\Phi$ is the constraint equations, $r$ is the application point of force, $f$ is the function defining the forces, $\mathrm{p}$ is the momentum, $\mathrm{u}$ is the velocity, $\mathrm{q}$ is the displacement, $\lambda$ is the Lagrange Multiplier, and $\mathrm{F}$ is the externally applied force.

At time $t_{n}$, the position of parts are calculated by Newton Raphon iteration using Equation (8).

$$
\left.\frac{\partial \Phi}{\partial q}\right|_{j} \Delta q_{j}=-\Phi\left(q_{i}, t_{n}\right)
$$

where $\nabla q_{j}=q_{j+1}-q_{j}, j$ denotes the $j$-th iteration.

Solving the first derivative and the second derivative of restriction Equation (6), instant velocity and acceleration at $t_{n}[10]$ are given by Equations (9) and (11).

$$
\begin{gathered}
\left(\frac{\partial \Phi}{\partial q}\right) \dot{q}=-\frac{\partial \Phi}{\partial t} \\
\left(\frac{\partial \Phi}{\partial q}\right) \ddot{q} \\
=-\left\{\frac{\partial^{2} \Phi}{\partial t^{2}}+\sum_{k=1}^{n} \sum_{l=1}^{n} \frac{\partial^{2} \Phi}{\partial q_{k} \partial q_{l}} \dot{q}_{k} \dot{q}_{l}+\frac{\partial}{\partial t}\left(\frac{\partial \Phi}{\partial q}\right) \dot{q}+\frac{\partial}{\partial q}\left(\frac{\partial \Phi}{\partial t}\right) \dot{q}\right\}
\end{gathered}
$$

From the Lagrange equation with multiplier, the instant reaction forces in the PWA multi-body system are given by Equation (11) along each of generalized coordinates. These are introduced as holonomic algebraic constraint functions. Therefore, the assembly of parts can be represented mathematically in a manner that conforms to the required dynamic functions of the system.

$$
\left(\frac{\partial \Phi}{\partial q}\right)^{T} \lambda=\left[-\frac{\mathrm{d}}{\mathrm{d} t}\left(\frac{\partial T}{\partial \dot{q}}\right)^{T}+\left(\frac{\partial T}{\partial q}\right)^{T}+f\right]
$$

\subsection{Static Model of the PWA Pipeline}

The static model of pipeline will be developed using the governing equation for freely hanging flexible cable [11] in order to compare the static tension with the dynamic tension of pipe. In the PWA system, the pipes between two wagons are freely handing pipes. In the following, the Equation [11] are presented for freely hanging pipes when the load is distributed uniformly along the pipes, as shown in Figure 3. The supports are both at same level. The span is L from lift-hand support to right-hand support. The position of maximum pipe sag $d$ occurs when $x$ $=\mathrm{L} / 2$. When pipe of length $\mathrm{L}$ subjected to a load $\mathrm{w} \times \mathrm{L}$, where $\mathrm{w}$ is the intensity of the distributed load along the horizontal projection of the pipe at any distance $x$ from the left-hand support, the tension, $T_{x}$, in the pipe with a distance $x$ from the left-hand support can be found from Equation (12). 


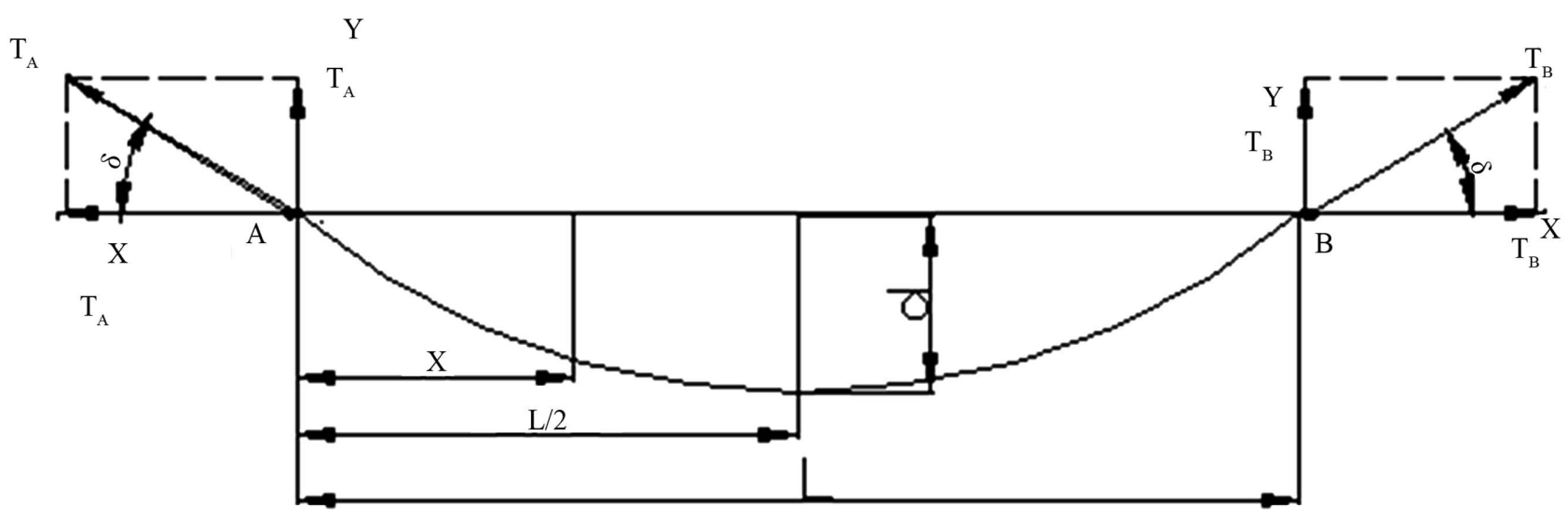

Figure 3. Simply supported pipe with uniformly distributed load along the horizontal projection of the span.

$$
T_{x}=H\left\{1+\left(\frac{4 d}{L^{2}}(L-2 x)\right)^{2}\right\}^{1 / 2}
$$

where $\mathrm{H}=\mathrm{wL}^{2} / 8 \mathrm{~d}$.

From Equation (12) it follows that the pipe tensions, $T_{A}$ and $T_{B}$, at the supports of $\mathrm{A}$ and $\mathrm{B}$ are given by

$$
T_{A}=T_{B}=H\left\{1+\left(\frac{4 \mathrm{~d}}{L}\right)^{2}\right\}^{1 / 2}
$$

The length of the pipe, $l$, can be expressed as

$$
l=L\left\{1+\frac{8}{3}\left(\frac{\mathrm{d}}{L}\right)^{2}-\frac{32}{5}\left(\frac{\mathrm{d}}{L}\right)^{4}\right\}
$$

The values of $\mathrm{H}, \mathrm{d}$ and $\mathrm{L}$ will change to $(\mathrm{H}+\Delta \mathrm{H})$, $(\mathrm{d}$ $+\Delta \mathrm{d})$ and $(\mathrm{L}+\Delta \mathrm{L})$ due to horizontal movements of the supports. If assumed that the length of the pipe does not alter due to changes in tension, the change in $\mathrm{d}$ may be found by differentiating Equation (14) with respect to L. This eventually yields

$$
\nabla \mathrm{d}=\frac{120 L^{4}-320 \mathrm{~d}^{2} L^{2}+2304 \mathrm{~d}^{4}}{640 \mathrm{~d} L^{3}-3072 \mathrm{~d}^{3} L} \nabla L
$$

\section{Virtual Prototype of the PWA System}

Figure 4 shows a virtual prototype of the PWA system developed in ADAMS environment. The PWA model, including the oil sands terrain, mobile slurry system, wagon subassembly, water line subassembly and slurry line subassembly, is modeled as a multi-body system. The design parameters for the PWA simulation will comprise basic dimension and calculation data as shown in Table 1. In this table, the flexible pipe system material of FlexRite is used to PWA pipeline material based on the conceptual design of the flexible pipeline system [1]. This flexible pipe can bend to a maximum degree of 60 . The specific gravities and pipeline diameters of slurry and water given in this table have been derived from Section 2. The unit length of pipeline and wagon listed in the table is assigned by the results of the PWA system synthesis. To accommodate a shovel advance of $400 \mathrm{~m}$ per week, the maximum displacement of every wagon is $7 \mathrm{~m}$. In order to operate the system, the drive force has to be applied to fourteen wagons, respectively. 3-step procedures for building the PWA model are described below. The first step involves the creation of 3-D component models of the oil sands terrain, mobile slurry system, wagon subassembly, water line subassembly and slurry line subassembly. The mobile slurry system contains one body and two crawler geometries. The wagon model includes one body and four crawler geometries. The water and slurry models consist of a series of smaller rigid pipe sections as show in Figure 4. The second step defines the connections of the components with joints. Figure 5 gives the topological structure and restriction among components of the PWA multi-body system. The oil sands terrain B0 and mobile slurry crawler B1 and wagon crawler B2 are connected by translational joints $\mathrm{H} 1$ and $\mathrm{H} 2$, respectively. The mobile slurry crawler B1 and mobile slurry body B3 are connected by revolute joint H3. The wagon crawler B2 and wagon body B4 are connected by revolute joint H4. The mobile slurry body B3 and water line B5 and slurry line B6 are connected by ball joints H5 and H6, respectively. The wagon body B4 and water line B5 and slurry line B6 are connected by ball joints $\mathrm{H} 5$ and H6, respectively. The flexible water pipeline B5 and slurry pipeline $\mathrm{B} 6$ are separated a lot of small sections that are connected by ball joint $\mathrm{H} 7$ and spring-damper H8, respectively. The third step defines the appropriate algebraic variables, which represent the movements of the mobile slurry system. This means that the varying angles applied to the water and slurry pipelines are introduced during operation. 
Table 1. Main PWA design parameters [1-3].

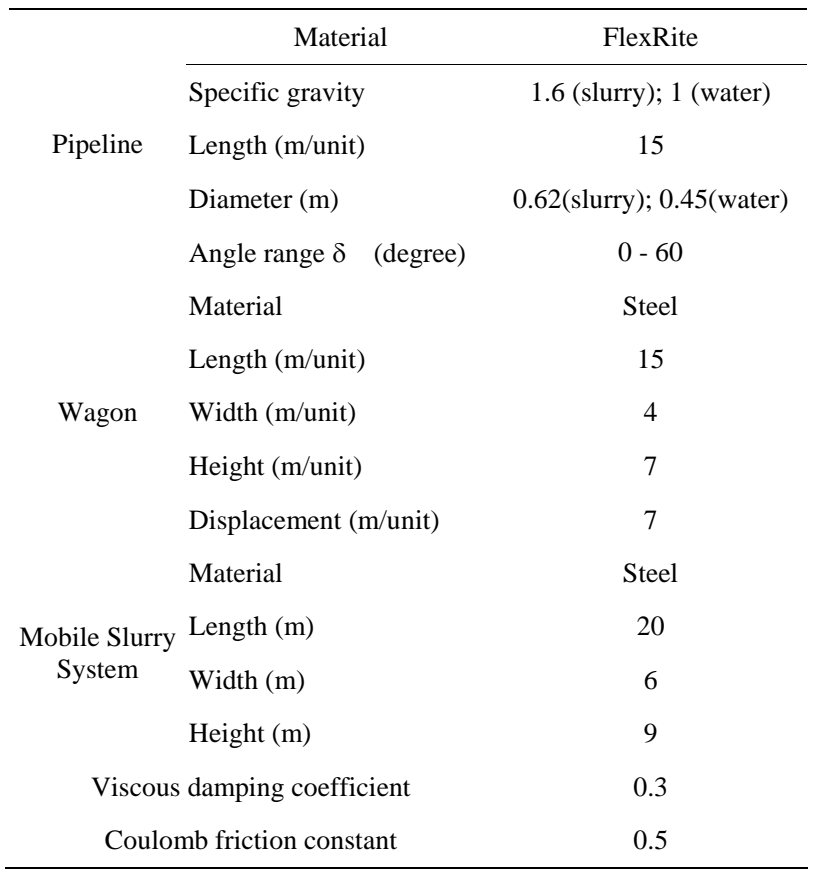

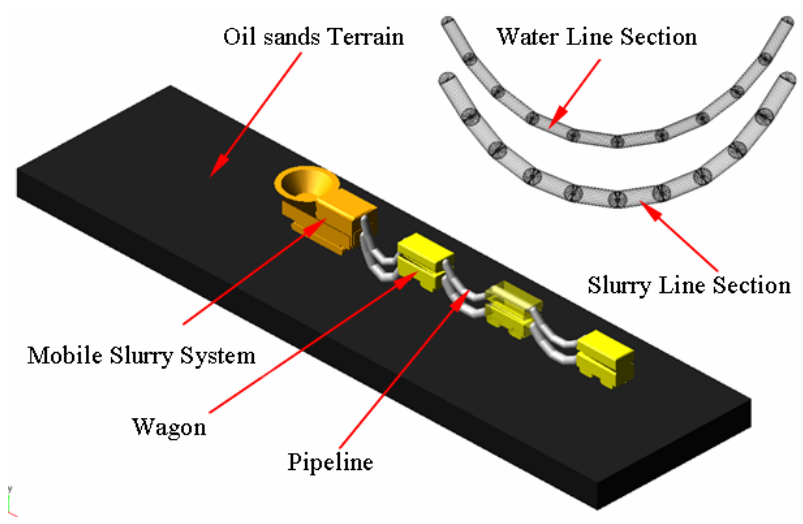

Figure 4. Virtual prototype of the PWA system.

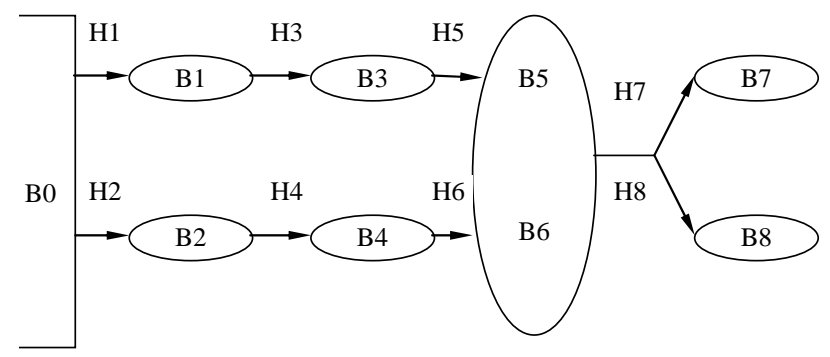

Figure 5. Topological and restriction among parts of the PWA system.

\section{Dynamic Simulation of the PWA System}

Visualizing system motion can be realized by simulating the PWA system. The PWA virtual prototype shown in
Figure 4 has been moved on a hard homogeneous oil sands terrain. In the 3-D solid model, the mass, inertial properties and gravity of components are given in Table 2 .

For one complete cycle of the PWA system, every wagon is commanded to execute the return and forward motions. The desired motion includes a return motion lasting $7 \mathrm{~m}$ within $500 \mathrm{~s}$ and a forward motion of $7 \mathrm{~m}$ during $500 \mathrm{~s}$, and corresponds to the every wagon velocity of 0.014 $\mathrm{m} / \mathrm{s}$. When static and dynamic friction coefficient between ground and crawler are set to 0.75 and 0.7 , respectively, the variations in response such as motion-generated forces, displacement of pipe wagon and angle $\delta$, have been obtained as measures of the system performance.

\subsection{Dynamic Motion Simulation of the PWA System}

Figure 6 shows that the motion of the PWA system is visualized by plotting successive six positions on graphic display over an extended period of time. The angles $\delta$, displacement of wagon and sag of pipe are plotted in Figures 7 (a) and (b), respectively.

Figure 7(a) shows the plot of angle $\delta$ (see Figure 3) versus time for one cycle. The angle $\delta=0^{\circ}$ at time $\mathrm{t}=0$ corresponds to the initial static equilibrium position. $\delta$ increases with time from 0 to $500 \mathrm{~s}$ followed by a decreases from 500 to $1000 \mathrm{~s}$. It undergoes a maximum of $56^{\circ}$ at time $=500 \mathrm{~s}$, which corresponds to the end position. The $\delta$ predicted from $0^{\circ}$ to $56^{\circ}$ for the system is in the range from $0^{\circ}$ to $60^{\circ}$ allowed by the pipe material.

Figure 7(b) depicts the displacement of every wagon and the sag of pipe for one cycle. Both displacement and sag increase with time for the first $500 \mathrm{~s}$ and they are subjected to the opposite trend for the last $500 \mathrm{~s}$. The maximum displacement locates at $7 \mathrm{~m}$ at a time of $500 \mathrm{~s}$, which meets the required value in Table 1 . The corresponding maximum sag of pipe is $6.8 \mathrm{~m}$.

\subsection{Dynamic Force Simulation of the PWA System}

The PWA prototype is simulated over an extended period of time to study dynamic-force of the system. The

Table 2. Mass and properties in the PWA system.

\begin{tabular}{lcccc}
\hline \multirow{2}{*}{ Component Name } & \multicolumn{4}{c}{ Inertia Moments $\left(\mathrm{kgm}^{2}\right)$} \\
\cline { 3 - 5 } & & Ixx & Iyy & Izz \\
\hline Water line (each section) & 480 & 12 & 138 & 138 \\
Slurry line (each section) & 928 & 42 & 308 & 308 \\
Wagon & $1.97 \mathrm{E}+5$ & $2.97 \mathrm{E}+5$ & $3.65 \mathrm{E}+6$ & $3.83 \mathrm{E}+6$ \\
Mobile Slurry & $6.55 \mathrm{E}+6$ & $4.64 \mathrm{E}+7$ & $2.45 \mathrm{E}+8$ & $2.38 \mathrm{E}+8$ \\
\hline
\end{tabular}




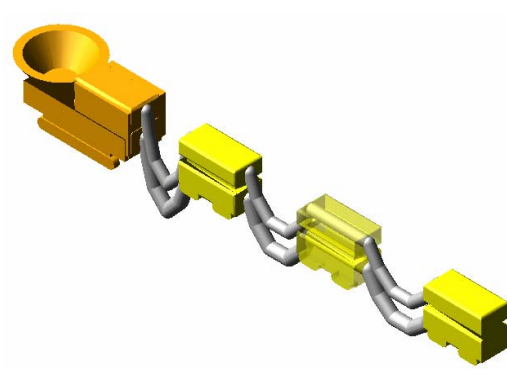

(a)

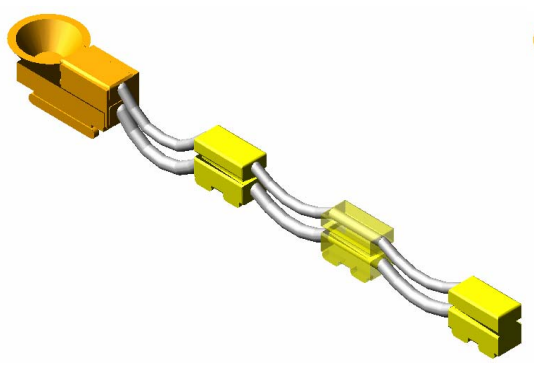

(d)

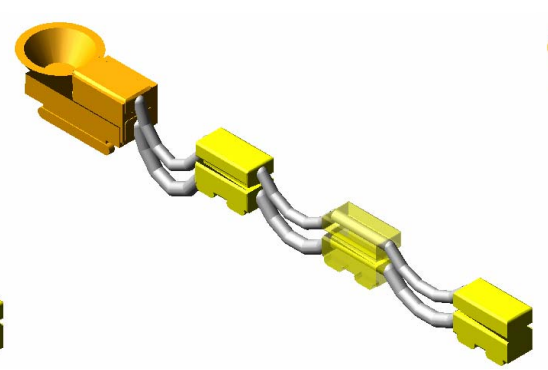

(b)

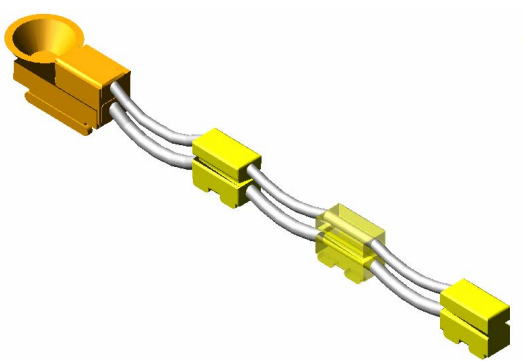

(e)

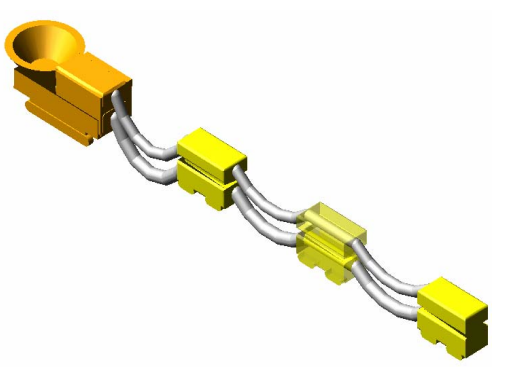

(c)

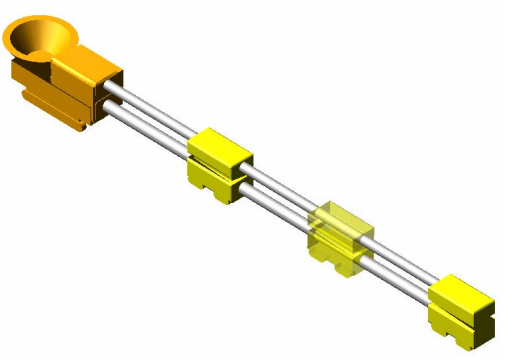

(f)

Figure 6. PWA motion sequence through one extended period of time.

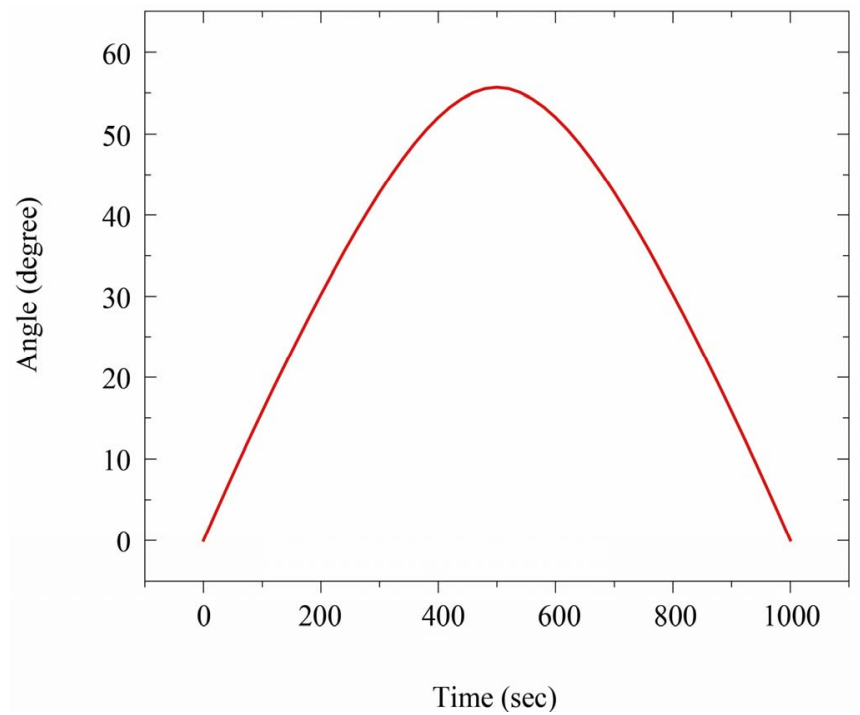

(a)

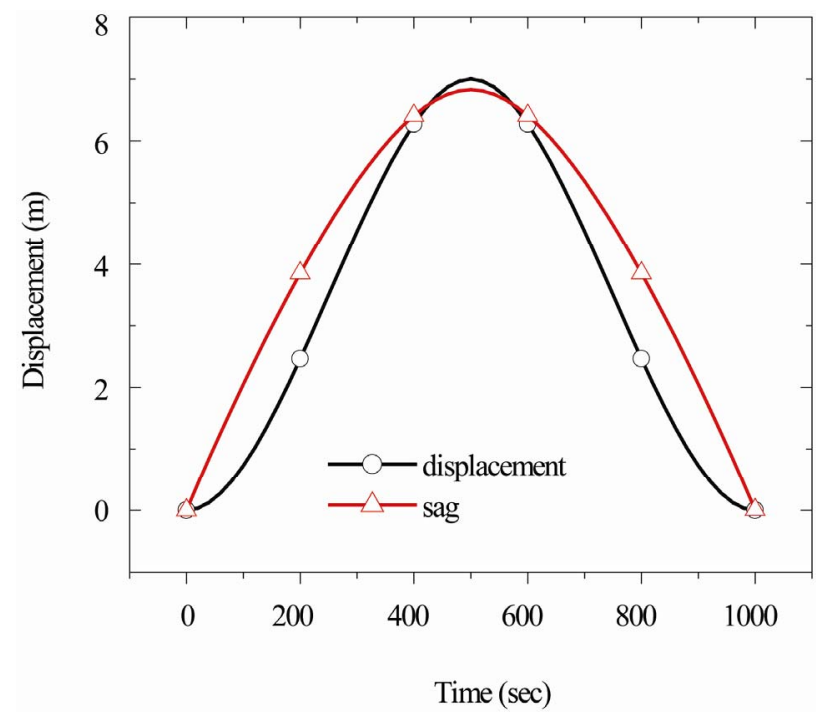

(b)

Figure 7. Dynamic motion responses to time for the PWA system: (a) angle and (b) displacement and sag.

driving force and non-driving force applied on wagon are plotted in Figures 8(a) and (b).

Figure 8(a) illustrates the driving force applied on each wagon for a wagon full motion cycle. It increases dramatically from 0 to ca. $1.5 \mathrm{E}+006 \mathrm{~N}$ at a time range from 0 to ca.100s. Then, a constant positive force of $1.5 \mathrm{E}+006 \mathrm{~N}$ occurs in the time range from 100 to ca. 500s. But, it dives sharply from $1.5 \mathrm{E}+006 \mathrm{~N}$ to ca. $-1.6 \mathrm{E}+006 \mathrm{~N}$ around a time of 500s. Finally, it decreases slowly to $-1.85 \mathrm{E}+$ $006 \mathrm{~N}$ between 500 and $1000 \mathrm{~s}$. The transient responses around time of 0 and $500 \mathrm{~s}$ disappear quickly. It should be noted that a positive force indicates retraction while a negative one does extension. This result indicates that the maximum driving force is a little smaller when system moves back than when it moves forth. The pipe gravity accounts for this difference. It has a positive effect on driving force when the wagon moves backward. However, it has a negative effect on driving force when the wagon moves forward.

Figure 8(b) displays the non-driving force applied on 


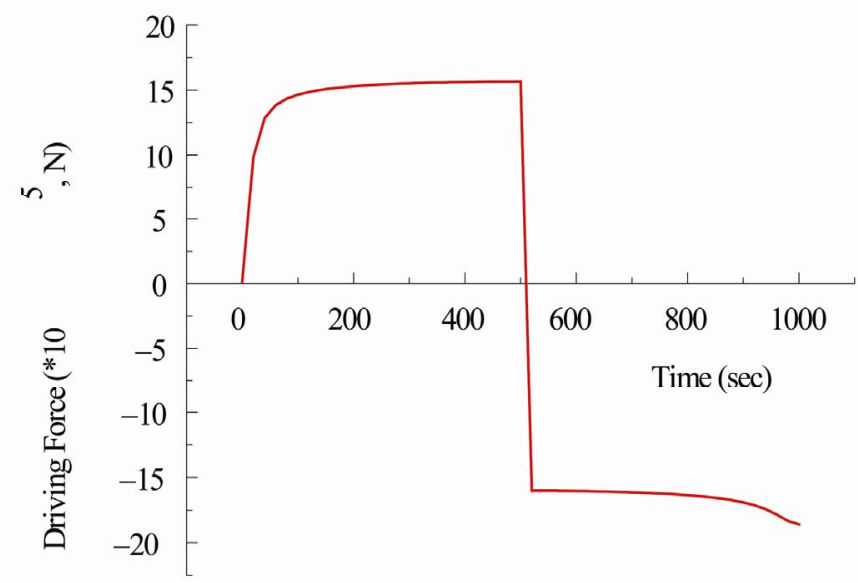

(a)

Figure 8. Dynamic force responses to time for the PWA system: (a) driving force applied on each wagon and (b) non-driving force applied on each wagon.

each wagon with time for one cycle. It goes down a little quickly from $-2.045 \mathrm{E}+006$ to $-2.1 \mathrm{E}+006 \mathrm{~N}$ around a time of $\mathrm{t}=0 \mathrm{~s}$, indicating a transient response dies down quickly around time $t=0$. Then a constant negative force of $-2.1 \mathrm{E}+006 \mathrm{~N}$ is established up to $1000 \mathrm{~s}$.

The tension of the slurry pipe is simulated by using the virtual prototype shown in Figure 4. The design parameters used here are given in Section 5. The wagon back or forth displacement of $7 \mathrm{~m}$ and the maximum pipe sag of $6.8 \mathrm{~m}$ have been derived from the dynamic motion tests above.

The relationship between displacement and dynamic tension is investigated here. Positions 1 - 6 with an interval of 1 are labeled by dividing a half pipe length from left-hand support to mid point (see Figure 9(a)). The corresponding tensions acting at these positions are shown in Figure 9(b) for a wagon movement from 0 to $7 \mathrm{~m}$. It is seen that the tension increases sharply from 0 to $4 \mathrm{E}+$ $005 \mathrm{~N}$ with displacement in a very small range from 0 to $0.3 \mathrm{~m}$ followed by a sharp decrease of the tension to ca. $1 \mathrm{E}+005 \mathrm{~N}$ within the displacement between 0.3 and $1 \mathrm{~m}$. Then, it decreases slowly with displacement. For a given displacement, tension decreases from positions 1 to 6 . The maximum tension appears at poison 1 (support) while the minimum one does at position 6 (the center of pipe).

The relationships of the displacement-static tension and the displacement-dynamic tension are discussed here. The variation of tension at support for a displacement from 0 to $7 \mathrm{~m}$ is shown in Figure 10. The static tension calculated by Equations (13)-(15) decreases slowly with displacement. By comparing the simulated dynamic tension with the static tension, it can be noted that the dynamic tension is greater than the static one owing to dynamic effect, especially before a stable tension is established.

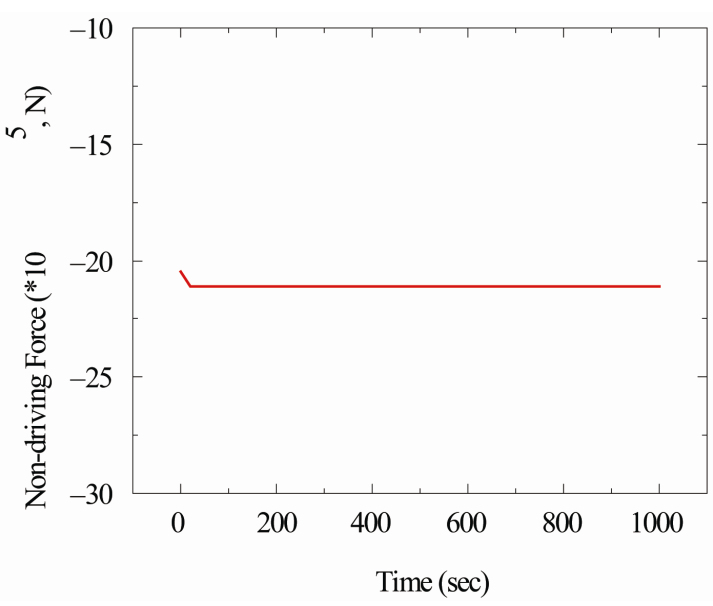

(b)

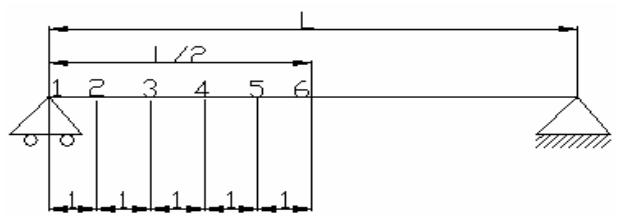

(a)

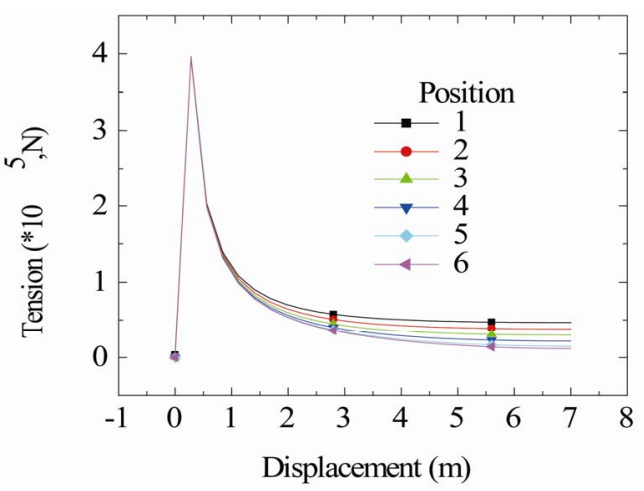

Figure 9. (a) Scheme of position distribution; (b) Displacement-dynamic tensions relationship with different positions.

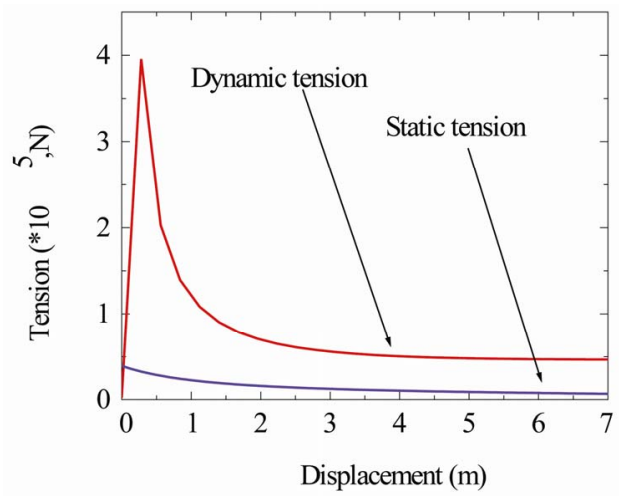

Figure 10. Static tension and dynamic tension at the support. 


\section{Conclusions}

The mechanical system of PWA has been simulated by using virtual model, which is developed by combining the theory of machines and mechanisms and the multi-body dynamic simulation software ADAMS. Important engineering data of the PWA system have been determined by simulating reality with a virtual prototype. The virtual prototype model of the PWA system has been tested and verified to be effective with real displacement value. The results show this model is capable of kinematics computing and offering computer-animated simulations of the kinematics behavior. The results of dynamic-motion analysis indicate that the conceptual designed the PWA system meets the requirement of the variation of angle $\delta$ from $0^{\circ}$ to $60^{\circ}$. The results of dynamic-force simulation have given the maximum force of $-1.85 \mathrm{E}+006 \mathrm{~N}$ for driving the system and the maximum non-driving force applied on wagon $(2.1 \mathrm{E}+006 \mathrm{~N})$ for calculating the bearing capacity of oil sands. The tension analysis of the pipe shows that the distribution of tension along the pipe length is not uniform. The maximum tension appears at the support. The result of comparison between the static tension and dynamic tension illustrates that static tension is much smaller than the dynamic tension. This work will allow further benchmarking of the mechanical event simulation of the PWA system such as examination of bearing capacity and prediction of pipeline stress.

\section{Acknowledgements}

The authors wish to express their gratitude to AERI/COURSE and Syncrude Canada Ltd. for the financial support and field data for this study.

\section{References}

[1] S. Frimpong, R. M. M. Changirwa, E. Asa and J. Szymanski, "Mechanics of Oil sands Slurry Flow in a Flexible Pipeline System,” International Journal of Surface
Mining, Vol. 16, No. 2, 2002, pp. 105-121. doi:10.1076/ijsm.16.2.105.3401

[2] S. Frimpong, O. R. Ayodele and J. Szymanski, "Numerical Simulation Software for Oil sands Slurry Flow in Flexible Pipelines," SCSC 2003 of the Society for Modeling and Simulation International, Montreal, 20-24 July 2003, pp. 145-154.

[3] S. Frimpong, O. R. Ayodele and J. Szymanski, "Numerical Simulator for Oil Sands Slurry Flow in Flexible Pipeline," In proceedings of the 2002 Summer Computer Simulation Conference, San Diego, 8-14 July 2002, pp. 171176.

[4] R. Changirwa, M. C. Rockwell, S. Frimpong and J. Szymanski, "Hybrid Simulation for Oil-Solids-Water Separation in Oil Sands Production," Minerals Engineering, Vol. 12, No. 12, 2002, pp. 1459-1468. doi:10.1016/S0892-6875(99)00134-X

[5] S. Frimpong, Y. Li and J. Szymanski, "Mechanical System Simulation of the Ground Articulating Pipeline System," Fifteenth IASTED International Conference on Modelling and Simulation, Marina Del Rey, 1-3 March 2004, pp. 209-213.

[6] J. E. Shigley and J. J. Uicker, "Theory of Machines and Mechanisms,” McGraw-Hill, New York, 1995.

[7] C. E. Wilson and J. P. Sadler, "Kinematics and Dynamics of Machinery,” 2nd Edition, Harper Collins College Publishers, New York, 1991.

[8] J. L. Synge and B. A. Griffith, "Principles of Mechanics," McGraw Hill Book Company, New York, 1959.

[9] C. W. Ham, E. J. Crank and W. L. Rogers, "Mechanics of Machinery,” McGraw-Hill, New York, 1958. N. X. Wu, Q. H. Sun, D. L. Yu and Y. A. Pan, "Kinematics Simulation and Application for Machine Tool Based on Multi-body System Theory," Journal of Southeast University, Vol. 20, No. 2, 2004, pp. 162-164.

[10] N. X. Wu, Q. H. Sun, D. L. Yu and Y. A. Pan, "Kinematics Simulation and Application for Machine Tool Based on Multi-body System Theory,” Journal of Southeast University, Vol. 20, No. 2, 2004, pp. 162-164.

[11] H. A. Buchholdt, “An Introduction to Cable Roof Structures,” Thomas Telford, London, 1999. 